\title{
Perioperative celecoxib administration for pain management after total knee arthroplasty - A randomized, controlled study
} Yu-Min Huang ${ }^{\dagger 1,2}$, Chiu-Meng Wang ${ }^{\dagger 3}$, Chen-Ti Wang ${ }^{\dagger 1}$, Wei-Peng Lin ${ }^{\dagger 1}$, Lih-Ching Horng ${ }^{4}$ and Ching-Chuan Jiang*1

\author{
Address: ${ }^{1}$ Department of Orthopaedic Surgery, National Taiwan University Department of Orthopaedic Surgery, National Taiwan University \\ Hospital, Taipei, Taiwan, ${ }^{2}$ Department of Orthopaedic Surgery, Shuang Ho Hospital of Taipei Medical University, Taipei, Taiwan, ${ }^{3}$ Department of \\ Orthopaedics, Kinmen Hospital Department of Health, Kinmen, Taiwan and ${ }^{4}$ Department of Nursing, College of Medicine, National Taiwan \\ University \& Hospital, Taipei, Taiwan \\ Email: Yu-Min Huang - yellowcorn0326@yahoo.com.hk; Chiu-Meng Wang - jormone1007@yahoo.com.tw; Chen- \\ Ti Wang - chentiwang@ntu.edu.tw; Wei-Peng Lin - nalew@pchome.com.tw; Lih-Ching Horng - lihli.tw@yahoo.com.tw; Ching- \\ Chuan Jiang* - ccj@ntu.edu.tw \\ * Corresponding author †Equal contributors
}

Published: 3 June 2008

BMC Musculoskeletal Disorders 2008, 9:77 doi:10.1 I86/147|-2474-9-77
Received: 17 December 2007

Accepted: 3 June 2008

This article is available from: http://www.biomedcentral.com/I47/-2474/9/77

(c) 2008 Huang et al; licensee BioMed Central Ltd.

This is an Open Access article distributed under the terms of the Creative Commons Attribution License (http://creativecommons.org/licenses/by/2.0), which permits unrestricted use, distribution, and reproduction in any medium, provided the original work is properly cited.

\begin{abstract}
Background: Non-steroidal anti-inflammatory drugs (NSAIDs) are recommended for multimodal postoperative pain management. We evaluated opioid-sparing effects and rehabilitative results after perioperative celecoxib administration for total knee arthroplasty.

Methods: This was a prospective, randomized, observer-blind control study. Eighty patients that underwent total knee arthroplasty were randomized into two groups of 40 each. The study group received a single $400 \mathrm{mg}$ dose of celecoxib, one hour before surgery, and $200 \mathrm{mg}$ of celecoxib every 12 hours for five days, along with patient-controlled analgesic (PCA) morphine. The control group received only PCA morphine for postoperative pain management. Visual analog scale (VAS) pain scores, active range of motion (ROM), total opioid use and postoperative nausea/vomiting were analyzed.

Results: Groups were comparable for age, pre-operative ROM, operation duration and intraoperative blood loss. Resting VAS pain scores improved significantly in the celecoxib group, compared with controls, at $48 \mathrm{hrs}(2.13 \pm \mathrm{I} .68$ vs. $3.43 \pm \mathrm{I} .50, \mathrm{p}=0.03)$ and $72 \mathrm{hrs}(\mathrm{I} .78 \pm \mathrm{I} .66$ vs. $3.17 \pm 2.0 \mathrm{I}, \mathrm{p}=0.02)$ after surgery. Active ROM also increased significantly in the patients that received celecoxib, especially in the first $72 \mathrm{hrs}\left[40.8^{\circ} \pm 17.3^{\circ}\right.$ vs. $25.8^{\circ} \pm 11.5^{\circ}, p=0.01$ (day I); $60.7^{\circ} \pm 18.1^{\circ}$ vs. $45.0^{\circ} \pm 17.3^{\circ}, p=0.004$ (day 2); $77.7^{\circ} \pm 15.1^{\circ}$ vs. $64.3^{\circ} \pm 16.9^{\circ}, p=0.004$ (day 3)]. Opioid requirements decreased about $40 \%(p=0.03)$ in the celecoxib group. Although patients suffering from post-operative nausea/vomiting decreased from $43 \%$ in control group to $28 \%$ in celecoxib group, this was not significant $(p=0.57)$. There were no differences in blood loss (intra- and postoperative) between the groups. Celecoxib resulted in no significant increase in the need for blood transfusions.
\end{abstract}

Conclusion: Perioperative celecoxib significantly improved postoperative resting pain scores at 48 and $72 \mathrm{hrs}$, opioid consumption, and active ROM in the first three days after total knee arthroplasty, without increasing the risks of bleeding.

Trial registration: Clinicaltrials.gov NCT00598234 


\section{Background}

Surgical trauma induces the synthesis of prostaglandins, which sensitize the peripheral nociceptors [1]. Non-steroidal anti-inflammatory drugs (NSAIDs) inhibit prostaglandin synthesis in the periphery and the spinal cord, therefore decreasing the post-operative hyperalgesic state [2]. NSAIDs have been shown to have opioid-sparing effects [3-6] and reduce postoperative nausea and vomiting (PONV) by 30\% [7]. However, the analgesic effects of perioperative NSAIDs are still uncertain [8-10]. Some studies suggest that perioperative NSAIDs improve postoperative pain for ambulatory arthroscopic knee [11] or spinal fusion surgery [12]. However, few papers have discussed the effectiveness of perioperative NSAID in pain management after total knee arthroplasty (TKA) [13-15]. Rofecoxib has been the perioperative coxib in previous studies. However, in September 2004, it was withdrawn from the market due to its thromboembolic effects, particularly myocardial infarction.

Celecoxib is a selective cyclooxygenase (COX)-2 inhibitor and an effective analgesic for acute postoperative pain [16]. Although pre-operative non-selective NSAID use increases the risks of bleeding [10,17], celecoxib $(1200$ mg daily) has no effects on serum thromboxane or platelet functions [16]. Celecoxib (400 mg) also has similar analgesic effects in comparison with conventional nonselective NSAID [4]. To achieve less postoperative pain and better rehabilitation after TKA surgery, especially in the first week, prescription of oral celecoxib preemptively for pain management of TKA patients is reasonable. Although previous studies have evaluated the analgesic efficacy of rofecoxib, few studies to date have evaluated the efficacy of celecoxib for TKA. In this study, we hypothesized that celecoxib provides better efficacy than the use of patient-controlled analgesic (PCA) morphine, which is currently the standard therapy in our institute. We aimed to compare the difference in the pain scores at rest and ambulation, along with range of motion (ROM), morphine-sparing effects, PONV, and perioperative blood loss between patients receiving celecoxib treatment and patients receiving PCA morphine treatment after TKA surgeries.

\section{Methods}

This study was performed (from September 2006 to March 2007) after institutional IRB approval. All TKA sur- geries were performed by one surgeon (Ching-Chuan Jiang). Under a randomized, prospective, observer-blind study design, subjects were sorted by random numbers into two groups. Inclusion criteria for this study were primary osteoarthritis and an age over sixty. Exclusion criteria for this study were rheumatoid arthritis, end-stage renal disease (complete kidney dysfunction requiring dialysis or kidney transplantation), previous cerebral vascular accident history, peptic ulcers, recent myocardial infarction (within 1 year), and allergy to sulf, NSAIDs, or morphine. The National Taiwan University Hospital and National Institutes of Health have approved the study and all participants will provide written informed consent.

The study group $(\mathrm{n}=40)$ received $400 \mathrm{mg}$ oral celecoxib at about $1 \mathrm{hr}$ prior to surgery, and $200 \mathrm{mg}$ every $12 \mathrm{hrs}$, along with PCA morphine, over the first five post-operative days. The control group $(n=40)$ received PCA morphine over the same postoperative period.

All patients had spinal anesthesia and hemovac drain tubes inserted for postoperative blood loss evaluation. Patient data included gender, age, range of motion (ROM), pain scores, blood loss and procedure duration. Pain scores were measured using a visual analog scale (VAS), with 0 indicating no pain and 10 indicating the worst imaginable pain [18]. Pain scores were checked by the same observer at rest at $6,12,24,48 \& 72 \mathrm{hrs}$ and 7 days after TKA surgery. We encouraged patients to ambulate 24 hrs after TKA. Pain scores at ambulation were also analyzed. We measured the range of motion before ambulation by using a goniometer. Patients were supine on the bed and we identified bony landmarks, including the greater trochanter, lateral femoral condyle, and the lateral mallelous, to facilitate goniometer placement. All ranges of motion were measured preoperatively and postoperatively by the same study nurse (Lih-Ching Horng). The CPM machine was used twice a day in all patients. PCA morphine dose and PONV occurrence were also recorded. All NSAIDs were discontinued at seven days after surgery. All patients received a patient-controlled analgesia (PCA) pump after surgery. Morphine $(1 \mathrm{mg} / \mathrm{mL})$ was given intravenously with increases of $2 \mathrm{~mL}$ and a lockout interval of 10 minutes. Additionaly, thromboembolic prophlaxis (oral aspirin $100 \mathrm{mg}$ ) was given for seven postoperative days in both groups.

Table I: Patient demographics and surgical data. There were no significant differences between these groups.

\begin{tabular}{lcccccc}
\hline Group & Age (yr) & $\begin{array}{c}\text { Pre-OP ROM } \\
\text { (degrees) }\end{array}$ & Pre-OP VAS & $\begin{array}{c}\text { Surgery Duration } \\
\text { (min) }\end{array}$ & $\begin{array}{c}\text { Tourniquet Duration } \\
\text { (min) }\end{array}$ & $\begin{array}{c}\text { Intra-operative blood } \\
\text { loss (ml) }\end{array}$ \\
\hline Control & $70 \pm 7$ & $118 \pm 17$ & $4.7 \pm 1.9$ & $75 \pm 17$ & $45 \pm 13$ & $177 \pm 54$ \\
Celecoxib & $70 \pm 7$ & $116 \pm 18$ & $5.1 \pm 1.7$ & $77 \pm 17$ & $46 \pm 17$ & $181 \pm 63$ \\
\hline
\end{tabular}


VAS Pain Score on Resting

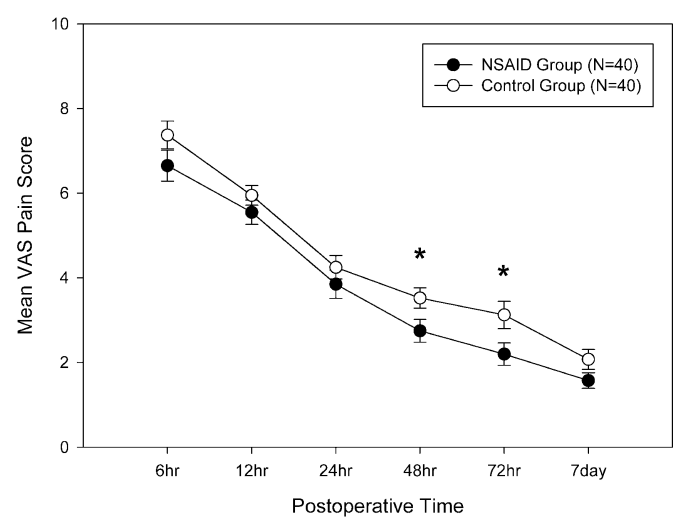

Figure I

Pain scores at rest. The celecoxib group had significantly less pain on Days 2 and 3. *: p value $<0.05$, and error bar indicates standard deviation.

The primary endpoint was VAS pain reduction through perioperative celecoxib administration. Group sample sizes of 40 and 40 can achieve $85 \%$ power in the detection of a difference of one point on a 10-point VAS scale, which is equivalent to the minimal clinical significant difference on the VAS scale [19], in a design with four repeated measurements when the standard deviation is 1.8 points. Group sample sizes of 40 and 40 can achieve $91 \%$ power in the detection of a difference of one point for a 10-point VAS scale in a design with six repeated measurements, when the standard deviation is 1.8 points. Secondary endpoints include ROM, morphine-sparing effects, postoperative nausea, vomiting and blood loss.

Patient demographics were analyzed by ANOVA or Chisquared test. Postoperative pain scores, postoperative ROM, morphine doses, blood loss, and PONV rates were analyzed by Mann-Whitney test. Group differences in pain intensity and ROM were also analyzed by repeated measures ANOVA test. Significance was defined as $\mathrm{p}<$ 0.05 .

\section{Results}

From Sep 2006 to March 2007, ninety-seven patients that fulfilled inclusion criteria received elective TKA surgery by the senior author (Ching-Chuan Jiang) at the author's institute. Fifteen patients were excluded (four with RA, three with ESRD, six with previous CVA, and two with a history of peptic ulcers). Two patients suffered from epigastralgia, without tarry stool, during the study and were also excluded, leaving a total of eighty patients. They were randomized into two groups. There were no significant
VAS Pain Score on Ambulation

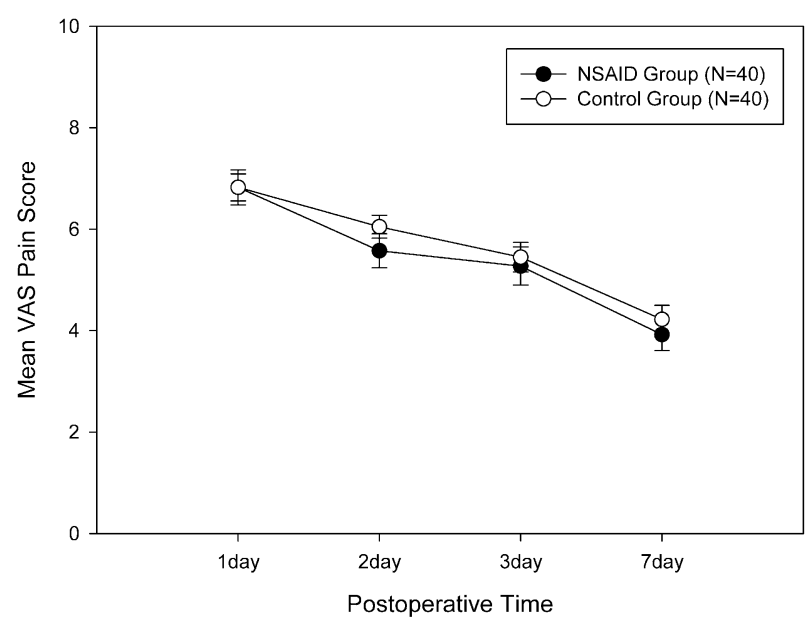

Figure 2

Pain scores at ambulation. There were no differences between the two groups. Error bar indicates standard deviation.

differences between them in age, preoperative ROM, preoperative pain scores, operation duration, and intraoperative blood loss (Table 1).

\section{VAS pain scores}

The celecoxib group showed less postoperative VAS pain at rest than the control group at 48 hrs $(p=0.03)$ and 72 hrs ( $p=0.02)$ after TKA surgery (Fig 1$)$. The celecoxib group had reduced postoperative pain at ambulation, but did not reach significant difference when compared with controls (Fig 2). Repeated measures ANOVA showed that the celecoxib group had significant VAS pain score reduction over the control group at rest $(\mathrm{p}=0.023)$ but not during ambulation $(\mathrm{p}=0.51)$.

\section{Range of motion}

Celecoxib significantly improved postoperative knee ROM, especially during the first three days (Day1: $\mathrm{p}=$ 0.01; Day 2: $\mathrm{p}=0.004$; Day 3: $\mathrm{p}=0.004$ ) (Fig 3). This group also had increased active ROM of 12-15 degrees in comparison with the control group. Repeated measures ANOVA showed that the celecoxib group also had significantly better postoperative ROM improvement than the control group $(\mathrm{p}=0.0009)$.

\section{Morphine-sparing effects and postoperative nausea and vomiting}

The celecoxib group had significantly less demand for PCA morphine usage (Fig 4). PCA usage during the first 24 hours was significantly lower $(15.1 \pm 8.7 \mathrm{mg}$ vs $19.7 \pm$ $9.6 \mathrm{mg} ; \mathrm{p}=0.03)$. Total PCA morphine usages in the 


\section{Active Range of Motion}

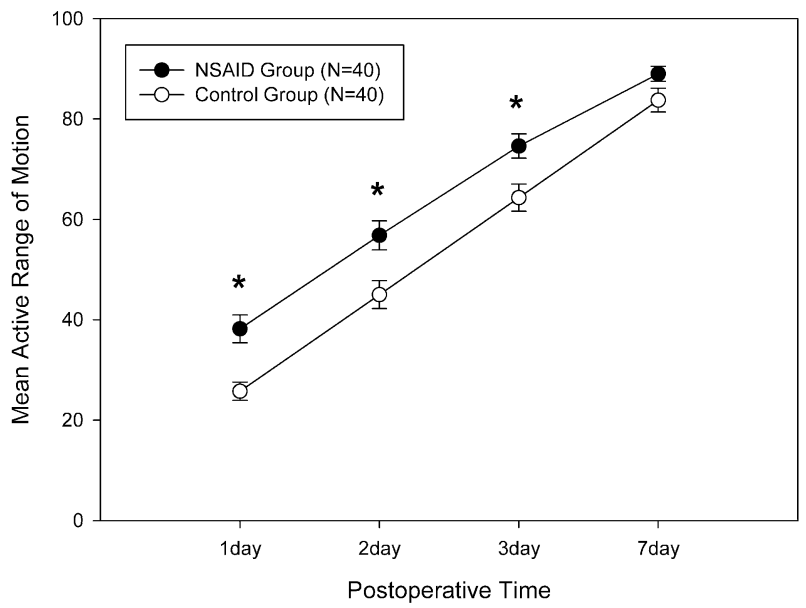

Figure 3

Results for the postoperative range of motion. The celecoxib group had significantly better active ROM during the first 3 days. $*$ : $p$ value $<0.05$, and error bar indicates standard deviation.

celecoxib group and controls were $17.6 \pm 11.9$ and $24.6 \pm$ $14.6 \mathrm{mg}$, respectively $(\mathrm{p}=0.03)$. The celecoxib group used about $40 \%$ less morphine. Postoperative nausea and vomiting were at $28 \%$ and $43 \%$ in the celecoxib group and controls, respectively. However, this did not reach statistical significance $(\mathrm{p}=0.57)$.

\section{Bleeding}

There were no significant differences in intraoperative blood loss (181 vs $177 \mathrm{ml}$ ), postoperative blood loss through Hemovac drain tubes (544 vs $511 \mathrm{ml}$ ), and postoperative blood transfusions (3.85 vs 3.93 units) between the celecoxib group and controls.

\section{Discussion}

In our study, we found that perioperative administration of celecoxib, a selective COX-2 inhibitor, reduced postoperative VAS pain scores at rest and decreased morphine usage while providing better ROM rehabilitation results. Perioperative administration of celecoxib did not increase perioperative bleeding or blood transfusion amounts.

Coxibs are effective analgesics for post-operative pain [2022]. To the best of our knowledge, the literature lacks a review of perioperative celecoxib for pain management in TKA patients. Perioperative coxibs have been shown to reduce postoperative pain and analgesic consumption while enhancing patient satisfaction [6]. In 2000, Reuben and Connelly studied perioperative use of celecoxib (200 $\mathrm{mg}$ ) and rofecoxib (50 mg) for spinal fusion surgery [12].
PCA Morphine Use

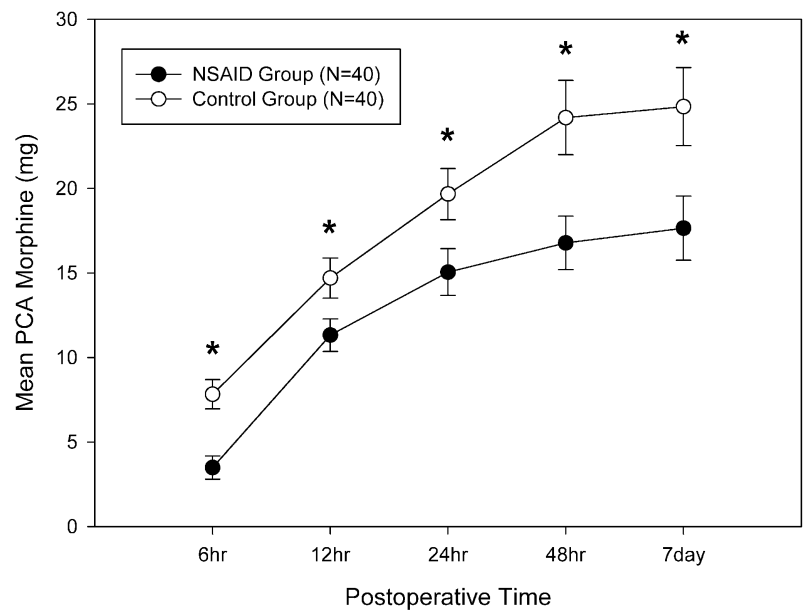

Figure 4

PCA morphine use. Mean PCA morphine use was significantly lower at each measurement for the celecoxib group. *: $P$ value $<0.05$, and error bar indicates standard deviation.

They concluded that both celecoxib and rofecoxib decrease morphine consumption, but that celecoxib's effects are limited. In their follow-up study, preoperative celecoxib $400 \mathrm{mg}$ showed better morphine-sparing effects than a single $200 \mathrm{mg}$ preoperative dose of celecoxib [23]. Therefore, we suggest that $400 \mathrm{mg}$ of celecoxib is more optimal than $200 \mathrm{mg}$ in controlling acute postoperative pain $[4,6]$. In our study, although VAS scores were significantly improved at 48 and 72 hrs at rest, and repeated measures ANOVA test also indicated that the celecoxib group had significantly lower pain scores at rest $(\mathrm{p}=$ 0.023), there was no significant difference between the two groups in pain scores at ambulation. We also demonstrate that perioperative celecoxib improves postoperative ROM results. The mean knee ROM was 92 degrees in the celecoxib group and 83.8 degrees in the control group, seven days after surgery.

NSAIDs are known to decrease opioid consumption by $30-50 \%[6,7,24]$. In our study, perioperative celecoxib significantly lowered it by $40 \%$. However, the effects of perioperative coxibs on opioid-related side effects are still uncertain [6]. This study also showed reduced postoperative nausea and vomiting ( $28 \%$ vs. $43 \%)$. However, there were no differences in PONV incidence. Further evaluation, using a larger sample, is called for.

Previous studies have shown that conventional preoperative non-selective NSAIDs increase bleeding risks $[17,25]$. Conventional non-selective NSAIDs reversibly inhibit COX and interfere with platelet functions, while selective 
COX-2 inhibitors have less antiplatelet effects than conventional non-selective NSAIDs $[26,27]$. In this study, perioperative administration of $400 \mathrm{mg}$ of celecoxib had no significant effects on blood loss, thus confirming indirectly that celecoxib has little effect on serum thromboxane or platelet functions [16]. The analgesic effects of aspirin may confuse the benefits of celecoxib, even though both groups are allowed to have aspirin in our study, and use of aspirin does not prevent the thromboembolic adverse effects of coxibs.

Selective COX-2 inhibitors may be a better choice for multimodal analgesia than conventional non-selective NSAID. Selective COX-2 inhibitors may have better gastrointestinal tolerance and less risk for cardiovascular events. It's suggested that selective COX-2 inhibitors have less gastrointestinal toxicity than conventional non-selective NSAIDs $[28,29]$. It's suggested that selective COX-2 inhibitors increase the risks of cardiovascular events, myocardial infarction, stroke and heart failure [29,30], too. White et al. also demonstrated no significant increase in $\mathrm{CV}$ risks with celecoxib in comparison to placebo or nonselective NSAIDs [31]. Moore et al. even demonstrated that celecoxib had less gastrointestinal and cardiovascular risk than conventional non-selective NSAIDs and all other coxibs [32]. However, there might not be any difference in the coxibs and conventional non-selective NSAIDs with respect to serious vascular events [33]. Selective COX-2 inhibitors may cause less bleeding than non-selective NSAID, because coxibs do not interfere the normal mechanisms of platelet aggregation and hemostasis, whereas non-selective NSAID produces significant reductions in platelet aggregation and serum thromboxane $B_{2}$ [34]. It has been shown that prior exposure of non-selective NSAID such as ibuprofen in THR surgery significant increases bleeding [10]. Therefore, we suggest that selective COX-2 is optimal for multimodal analgesia.

One limitation of this study is the lack of a true placebo group. Placebo response has been observed in up to $30 \%$ of patients that undergo surgery. However, placebo usage may present ethical concerns in the postoperative care of TKA surgery; we therefore adopted an active-control trial design that compared celebrex treatment to the standard therapy (PCA morphine) at our institute. Another limitation was the limited research period. We recorded data for only the first seven days. Although we demonstrated that celecoxib had better rehabilitative results in the first week than PCA morphine, long-term efficacy is still in question. There may be many reasons for the resting VAS scores to not have reached significance until 48 hours after surgery. One possibility is that the true difference does exist and that a larger sample size is needed to draw out the difference, despite that we had shown that the sample size in our study had adequate power.

\section{Conclusion}

Perioperative celecoxib, combined with opioids, is an effective and safe regimen for pain control in TKA patients. Perioperative celecoxib can significantly decrease postoperative pain scores at rest and opioid use, while improving postoperative ROM results for TKA surgeries. Considering the benefits, we suggest celecoxib for multimodal analgesia.

\section{Competing interests}

The authors declare that they have no competing interests.

\section{Authors' contributions}

$\mathrm{Y}-\mathrm{MH}, \mathrm{C}-\mathrm{MW}, \mathrm{W}-\mathrm{PL}$, and $\mathrm{C}-\mathrm{CJ}$ were responsible for the design of this study. $\mathrm{L}-\mathrm{CH}$ was the study evaluator and recorder. C-TW provided analytical support. Y-MH, CTW and C-CJ prepared the writing of the manuscript. All authors read and approved the final manuscript.

\section{Acknowledgements}

We thank the patients who participated in this study and the staff involved in this work. The study was supported by a research fund from the National Taiwan University \& Hospital.

\section{References}

I. Samad TA, Moore KA, Sapirstein A, Billet S, Allchorne A, Poole S, Bonventre JV, Woolf CJ: Interleukin- I beta-mediated induction of Cox-2 in the CNS contributes to inflammatory pain hypersensitivity. Nature 200 I, 4 I 0(6827):47| -475.

2. McCormack K: Non-steroidal anti-inflammatory drugs and spinal nociceptive processing. Pain 1994, 59(I):9-43.

3. Hyllested M, Jones S, Pedersen JL, Kehlet H: Comparative effect of paracetamol, NSAIDs or their combination in postoperative pain management: a qualitative review. British journal of anaesthesia 2002, 88(2): 199-2।4.

4. Romsing J, Moiniche S: A systematic review of COX-2 inhibitors compared with traditional NSAIDs, or different COX-2 inhibitors for post-operative pain. Acta anaesthesiologica Scandinavica 2004, 48(5):525-546.

5. Elia N, Lysakowski C, Tramer MR: Does multimodal analgesia with acetaminophen, nonsteroidal antiinflammatory drugs, or selective cyclooxygenase- 2 inhibitors and patient-controlled analgesia morphine offer advantages over morphine alone? Meta-analyses of randomized trials. Anesthesiology 2005, 103(6): 1296-1304.

6. Straube S, Derry S, McQuay HJ, Moore RA: Effect of preoperative Cox-II-selective NSAIDs (coxibs) on postoperative outcomes: a systematic review of randomized studies. Acta anaesthesiologica Scandinavica 2005, 49(5):60I-6I3.

7. Marret E, Kurdi O, Zufferey P, Bonnet F: Effects of nonsteroidal antiinflammatory drugs on patient-controlled analgesia morphine side effects: meta-analysis of randomized controlled trials. Anesthesiology 2005, I 02(6): I 249- 1260.

8. Huang JJ, Taguchi A, Hsu H, Andriole GL Jr, Kurz A: Preoperative oral rofecoxib does not decrease postoperative pain or morphine consumption in patients after radical prostatectomy: a prospective, randomized, double-blinded, placebo-controlled trial. J Clin Anesth 200I, I3(2):94-97.

9. Moiniche $S$, Kehlet H, Dahl JB: A qualitative and quantitative systematic review of preemptive analgesia for postoperative pain relief: the role of timing of analgesia. Anesthesiology 2002 , 96(3):725-74I.

10. Bugter ML, Dirksen R, Jhamandas K, Slappendel R, Weber EW, Milne $B$ : Prior ibuprofen exposure does not augment opioid drug potency or modify opioid requirements for pain inhibition in total hip surgery. Can J Anaesth 2003, 50(5):445-449.

II. Reuben SS, Bhopatkar S, Maciolek H, Joshi W, Sklar J: The preemptive analgesic effect of rofecoxib after ambulatory arthro- 
scopic knee surgery. Anesthesia and analgesia 2002, 94(I):55-59. table of contents

12. Reuben SS, Connelly NR: Postoperative analgesic effects of celecoxib or rofecoxib after spinal fusion surgery. Anesthesia and analgesia 2000, 9 I(5): I22I-I 225.

13. Reuben SS, Fingeroth R, Krushell R, Maciolek H: Evaluation of the safety and efficacy of the perioperative administration of rofecoxib for total knee arthroplasty. J Arthroplasty 2002, I 7(I):26-3I.

14. Buvanendran A, Kroin JS, Tuman KJ, Lubenow TR, Elmofty D, Moric $M$, Rosenberg AG: Effects of perioperative administration of a selective cyclooxygenase 2 inhibitor on pain management and recovery of function after knee replacement: a randomized controlled trial. Jama 2003, 290(I8):24| I-24I8.

15. Reuben SS, Buvenandran A, Katz B, Kroin JS: A prospective randomized trial on the role of perioperative celecoxib administration for total knee arthroplasty: improving clinical outcomes. Anesth Analg 2008, 106(4):1258-1264. table of contents

16. Clemett $D$, Goa KL: Celecoxib: a review of its use in osteoarthritis, rheumatoid arthritis and acute pain. Drugs 2000, 59(4):957-980.

17. Marret E, Flahault A, Samama CM, Bonnet F: Effects of postoperative, nonsteroidal, antiinflammatory drugs on bleeding risk after tonsillectomy: meta-analysis of randomized, controlled trials. Anesthesiology 2003, 98(6): I 497-I502.

18. Sriwatanakul K, Kelvie W, Lasagna L, Calimlim JF, Weis OF, Mehta G: Studies with different types of visual analog scales for measurement of pain. Clin Pharmacol Ther 1983, 34(2):234-239.

19. Kelly AM: The minimum clinically significant difference in visual analogue scale pain score does not differ with severity of pain. Emerg Med J 200I, I 8(3):205-207.

20. Barden J, Edwards JE, McQuay HJ, Moore RA: Single-dose rofecoxib for acute postoperative pain in adults: a quantitative systematic review. BMC Anesthesiol 2002, 2(I):4.

21. Barden J, Edwards JE, McQuay HJ, Moore RA: Oral valdecoxib and injected parecoxib for acute postoperative pain: a quantitative systematic review. BMC Anesthesiol 2003, 3(I): I.

22. Edwards JE, Moore RA, McQuay HJ: Individual patient meta-analysis of single-dose rofecoxib in postoperative pain. $B M C$ Anesthesiol 2004, 4(I):3.

23. Reuben SS, Ekman EF: The effect of cyclooxygenase-2 inhibition on analgesia and spinal fusion. The Journal of bone and joint surgery 2005, 87:536-542.

24. Romsing J, Moiniche S, Mathiesen O, Dahl JB: Reduction of opioidrelated adverse events using opioid-sparing analgesia with COX-2 inhibitors lacks documentation: a systematic review. Acta anaesthesiologica Scandinavica 2005, 49(2): I33-I 42.

25. Rogers JE, Fleming BG, Macintosh KC, Johnston B, Morgan-Hughes JO: Effect of timing of ketorolac administration on patientcontrolled opioid use. British journal of anaesthesia 1995, 75(I): $15-18$.

26. Klein T, Nusing RM, Pfeilschifter J, Ullrich V: Selective inhibition of cyclooxygenase 2. Biochem Pharmacol 1994, 48(8): |605-1610.

27. Riendeau D, Percival MD, Boyce S, Brideau C, Charleson S, Cromlish W, Ethier D, Evans J, Falgueyret JP, Ford-Hutchinson AW, Gordon R, Greig G, Gresser M, Guay J, Kargman S, Leger S, Mancini JA, O'Neill G, Ouellet M, Rodger IW, Therien M, Wang Z, Webb JK, Wong E, Chan CC: Biochemical and pharmacological profile of a tetrasubstituted furanone as a highly selective COX-2 inhibitor. Br J Pharmacol 1997, I 2 I(I): 105-117.

28. Bombardier C, Laine L, Reicin A, Shapiro D, Burgos-Vargas R, Davis B, Day R, Ferraz MB, Hawkey CJ, Hochberg MC, Kvien TK, Schnitzer $\mathrm{TJ}$ : Comparison of upper gastrointestinal toxicity of rofecoxib and naproxen in patients with rheumatoid arthritis. VIGOR Study Group. N EnglJ Med 2000, 343(2 I): I520-I528. 1522 p following 1528

29. Silverstein FE, Faich G, Goldstein JL, Simon LS, Pincus T, Whelton A Makuch R, Eisen G, Agrawal NM, Stenson WF, Burr AM, Zhao WW, Kent JD, Lefowith JB, Verburg KM, Geis GS: Gastrointestinal toxicity with celecoxib vs nonsteroidal anti-inflammatory drugs for osteoarthritis and rheumatoid arthritis: the CLASS study: A randomized controlled trial. Celecoxib Long-term Arthritis Safety Study. Jama 2000, 284(10): 1247-1255.

30. Solomon SD, McMurray JJ, Pfeffer MA, Wittes J, Fowler R, Finn P, Anderson WF, Zauber A, Hawk E, Bertagnolli M: Cardiovascular risk associated with celecoxib in a clinical trial for colorectal adenoma prevention. N Engl J Med 2005, 352(II): I07I-I080.

3I. White WB, West CR, Borer JS, Gorelick PB, Lavange L, Pan SX, Weiner E, Verburg KM: Risk of cardiovascular events in patients receiving celecoxib: a meta-analysis of randomized clinical trials. Am J Cardiol 2007, 99(I):91-98.

32. Moore RA, Derry S, McQuay HJ: Cyclo-oxygenase-2 selective inhibitors and nonsteroidal anti-inflammatory drugs: balancing gastrointestinal and cardiovascular risk. BMC Musculoskelet Disord 2007, 8:73.

33. Kearney PM, Baigent C, Godwin J, Halls H, Emberson JR, Patrono C: Do selective cyclo-oxygenase-2 inhibitors and traditional non-steroidal anti-inflammatory drugs increase the risk of atherothrombosis? Meta-analysis of randomised trials. $B M$ (Clinical research ed) 2006, 332(7553): I302-1308.

34. Leese PT, Hubbard RC, Karim A, Isakson PC, Yu SS, Geis GS: Effects of celecoxib, a novel cyclooxygenase-2 inhibitor, on platelet function in healthy adults: a randomized, controlled trial. Journal of clinical pharmacology 2000, 40(2): 124-132.

\section{Pre-publication history}

The pre-publication history for this paper can be accessed here:

http://www.biomedcentral.com/1471-2474/9/77/prepub

Publish with Biomed Central and every scientist can read your work free of charge

"BioMed Central will be the most significant development for disseminating the results of biomedical research in our lifetime. "

Sir Paul Nurse, Cancer Research UK

Your research papers will be:

- available free of charge to the entire biomedical community

- peer reviewed and published immediately upon acceptance

- cited in PubMed and archived on PubMed Central

- yours - you keep the copyright 